\title{
$\mu$-Holomorphic Projective Connections and Conformal Covariance
}

\author{
Mohamed KACHKACHI
}

Unité de Physique Mathématique, Département de Mathématiques et Informatique, Faculté des Sciences et Techniques, Université Hassan $1^{e r}$, Settat, Morocco ${ }^{1}$

The Abdus Salam International Centre for Theoretical Physics, Trieste, Italy

and

Lab/PHE, Departement de Physique, Facult des Sciences, Universit MV, Rabat, Morocco

Received December 20, 2002; Accepted May 22, 2003

\begin{abstract}
At the quantum level of a bidimensional conformal model, the conformal symmetry is broken by the diffeomorphism anomaly and the conformal covariance is not maintained. Here we interpret geometrically this conformal covariance as an exact $\mu$ holomorphy condition on a two-dimensional Riemann surface $\Sigma$ on which the model is constructed. On the other hand, to restore this conformal covariance, a holomorphic projective connection is needed. Here we get its transformation law with respect to a quasiconformal transformation on any complex Riemann surface and we recover the transformation law of a holomorphic projective connection under a holomorphic change of coordinates.
\end{abstract}

\section{$1 \mu$-Holomorphy conditions}

Two-dimensional conformal field theories on Riemann surfaces without boundaries are relevant models in string theory [1]. The dependence of these bidimensional conformal models on the background geometry turns out to be useful for the construction of effective actions for two-dimensional gravity [2]. On the other hand this geometrical dependence is well exhibited using the Beltrami parametrization of the bidimmensional world sheet metric of the bosonic string [3]. Moreover Beltrami differentials parametrize conformal classes of the metric on a Riemann surface $\Sigma$ on which the two-dimensional conformal model is constructed and satisfy the ellipticity condition: $S u p_{\Sigma}|\mu| \prec 1, \mu \in C^{\infty}(\Sigma)$. If we consider a complex structure $(z, \bar{z})$ the conformal classes of the metric $g$ are characterized by $d s^{2}=g_{\alpha \beta} \mathrm{d} x^{\alpha} d x^{\beta}=\rho^{2}|d z+\mu d \bar{z}|^{2}$ where $\rho(z, \bar{z})$ is the conformal factor. Indeed It is the most natural parametrization which exhibits the holomorphic factorization of Green's functions. Moreover, in the framework of this geometrical formulation, the degree of freedom of the Weyl symmetry is eliminated from the very begining. The remaining diffeomorphism symmetry is kept as the basic local invariance of the string action [3].

Copyright (c) 2004 by M Kachkachi

${ }^{1}$ Permanent address, 
However, at the quantum level, this diffeomorphism symmetry is broken and an anomaly emerges in this way the strength of which is measured by the central charge of the model. On the other hand each complex structure of the Riemann surface on which the model is constructed is associated with a projective structure that is parametrized by a projective connection. This latter is required to preserve the conformal covariance of the above mentioned diffeomorphism anomaly that appears in the quantum Ward identity (see [4] and references therein):

$$
W_{2} \frac{\delta \Gamma_{W Z P}[\mu]}{\delta \mu}=\frac{k}{12 \pi} L_{3}^{R}(\mu)
$$

where $W_{2} \equiv \bar{\partial}-\mu \partial-2 \partial \mu$ is the well-known Ward operator, $k$ is the central charge of the model under consideration and $\Gamma_{W Z P}$ is the Wess-Zumino-Polyakov effective action of the two-dimensional conformal model which is given by

$$
\Gamma_{W Z P}[\mu]=\frac{-k}{24 \pi} \int_{C}\left[d m \mu \partial^{2} \ln \partial Z\right](z)
$$

such that $d m(z, \bar{z}) \equiv d \bar{z} \wedge d z / 2 i$ is the two-dimensional measure on the complex plane and the scalar field $Z$ (called the Wess-Zumino field) satisfies the $\mu$-holomorphy condition (1.4) (see below). $L_{3}^{R} \equiv \partial^{3}+2 R \partial+\partial R$, the conformally covariant form of the operator $\partial^{3}$, is the third Bol's operator associated to the projective connection R.

First let me consider a bidimensional Riemann surface $\Sigma$ without boundary equipped with an atlas of compatible analytic systems of coordinates, i.e. we have complex coordinates $z_{\alpha}$ defined on each patch and the transition functions $h_{\alpha \beta}$ between two patches are holomorphic:

$$
z_{\alpha}=h_{\alpha \beta}\left(z_{\beta}\right) .
$$

In the case of the non holomorphic transition functions the two complex structures $\left(z_{\alpha}\right)$ and $\left(z_{\beta}\right)$ belong to different atlases. Then a Riemann surface $\Sigma$ is a $C^{\infty}$-differentiable twodimensional manifold endowed with a reference complex structure $(z, \bar{z})$ ( in the sense that $(z, \bar{z})$ is associated to the Beltrami differential $\mu(z, \bar{z})=0)$. On this surface we consider a Beltrami differential $\mu(z, \bar{z}) \neq 0$ that induces another complex structure $(Z, \bar{Z})$ on the surface $\Sigma$. These latter coordinates are $C^{\infty}$-diffeomorphisms of the reference variables $(z, \bar{z})$ and satisfy the following Beltrami equations:

$$
\begin{aligned}
& W_{0} Z=0, \\
& \overline{W_{0} Z}=0
\end{aligned}
$$

where $\mu \in C^{\infty}(\Sigma),|\mu| \prec 1$ and $W_{0} \equiv \bar{\partial}-\mu \partial$. This equation is defined up to a conformal transformation. Indeed, as one can verfy, for any function $F$ on the new complex structure, $F(Z)$, we have:

$$
W_{0} F=0 .
$$

Furthermore equation (1.5) is equivalent to the holomorphic condition for the function $F$ in the new complex structure $(Z, \bar{Z})$ :

$$
\partial_{\bar{Z}} F=0
$$


where $\partial_{\bar{Z}} \equiv \partial / \partial \bar{Z}$. Then the diffeomorphism

$$
z \rightarrow Z(z, \bar{z}), \bar{\partial} Z=\mu \partial Z
$$

is called a quasiconformal transformation the dilatation of which is the Beltrami differential $\mu$ (see [5] for details)This transformation, as one can see, becomes conformal for $\mu=0$.

Now we consider a vector bundle on the surface $\Sigma$ the base manifold of which is the set of all conformal structures and the fibre of which at a given conformal structure $\mu$ is a complex vector space of $\mu$-holomorphic j-differentials $f_{j}$ which depend holomorphically on the Beltrami differential $\mu$ (see below), i.e.

$$
\frac{\delta f_{j}[\mu]}{\delta \mu}
$$

These $\mu$-holomorphic j-differentials $f_{j}(z, \bar{z})$, defined on any reference complex structure $(z, \bar{z})$ of the complex surface $\Sigma$, satisfy the following $\mu$-holomorphy condition [2]:

$$
W_{j} f_{j}=0
$$

where $W_{j} \equiv \bar{\partial}-\mu \partial-j \partial \mu$ is a generalized Ward operator. More generally a collection $\eta$ of functions $\eta_{\alpha}$ defined on each domain $D_{\alpha}$ by

$$
\eta_{\alpha}: D_{\alpha} \rightarrow \Sigma, \eta_{\alpha}=\eta \circ z_{\alpha}
$$

is called a $(p, q)$-differential on $\Sigma$ if it is invariant under a holomorphic changes of coordinates: $\left(D_{\alpha}, z_{\alpha}\right) \rightarrow\left(D_{\beta}, z_{\beta}\right)$ an it is written locally as

$$
\eta=\eta_{\alpha \beta}(z, \bar{z}) d z^{p} d \bar{z}^{q}
$$

The Beltrami differential $\mu$, a aprticular case of $(1.11)$, is a $(-1,1)$-differential: $\mu \equiv$ $\mu_{\bar{z}}^{z} d \bar{z} \otimes \partial$ (see [3] and [5]. On the other hand, as one can verify, the particular case for $\mathrm{j}=0$ of (1.9) is the Beltrami equation (1.4), where $Z$ is viewed as a 0 -differential in the reference complex structure $(z, \bar{z})$. Moreover a solution of the general $\mu$-holomorpy condition (1.9) is given by $[6]$ :

$$
f_{j}=\lambda^{j} F
$$

where $F$ satisfies equation (1.5) and $\lambda \equiv \partial Z$ is called the conformal factor.

\section{Conformal Ward identities}

As a physical application the classical Ward identity of an effective two-dimensional conformal model, in the Beltrami parametrization scheme, is the $\mu$-holomorphy condition of the classical energy-momentum tensor $\Theta_{z z}(z, \bar{z}) \equiv \delta S_{C} / \delta \mu\left(S_{C}\right.$ is the classical effective action of the model) [3]:

$$
W_{2} \Theta_{z z}=0
$$


As we can verify this latter equation is the $\mathrm{j}=2$ particular case of the general $\mu$-holomorpy condition (1.9). At the quantum level a diffeomorphism anomaly occurs and the above Ward identity is translated to the anomalous one:

$$
W_{2} T_{z z}=\partial^{3} \mu
$$

where $T_{z z} \equiv \delta Z_{v}^{c} / \delta \mu$ is the quantum effective energy-momentum tensor of the model under consideration, $\partial^{3} \mu$ is the diffeomorphism anomaly and $Z_{v}^{c}$ is the connected generating Green's functions of the vaccum. Geometrically speaking the quantum Ward identity can be interpreted as a deformed $\mu$-holomorphy condition by the term $\partial^{3} \mu$. Indeed $T_{z z}$ is not a tensor (precisely not a 2-differential a $(2,0)$-differential following the definition of $\mathrm{j}$-differentials introduced before) under a holomorphic change of coordinates; $z \rightarrow \omega(z, \bar{z})$ :

$$
T_{z z} \rightarrow T_{\omega \omega}=(\partial \omega)^{-2}\left(T_{z z}-\zeta_{z}(\omega)\right),
$$

where $\zeta_{z}(\omega) \equiv \partial^{2} \ln \partial \omega-\frac{1}{2}(\partial \ln \omega)^{2}$ is the Schwarzian derivative of the diffeomorphism $z \rightarrow \omega(z, \bar{z})$ (see [3] and [5]). It is clear that a such a deformation comes from the diffeomorphism anomaly. On the other hand, taking into acount the Polyakov conjecture for a two-dimensional conformal model on the complex plane [3], the action (1.2) can be rewritten as the sum of the Neumann series $Z_{v}^{c}[\mu]$ :

$$
\Gamma_{W Z P}[\mu]=\frac{-k}{24 \pi} Z_{v}^{c}[\mu]
$$

Then it is easy to get:

$$
\frac{\delta Z_{v}^{c}[\mu]}{\delta \mu}=\zeta_{z}(Z)
$$

where $\zeta_{z}(Z)$ is the Schwarzian derivative of the quasiconformal transformation (1.7) which satisfies the following deformed $\mu$-holomorphy condition:

$$
W_{2} \zeta_{z}(Z)=\partial^{3} \mu
$$

This latter can be rewritten in conformally covariant form as

$$
\bar{\partial} \zeta_{z}(Z)=L_{3}^{\zeta_{z}(Z)}(\mu)
$$

Moreover the combination of equations (2.2) and (2.6) shows that $f_{z z} \equiv T_{z z}-\zeta_{z}(Z)$ is a 2 -differential which satisfies the following exact $\mu$-holomorphy condition:

$$
W_{2} f_{z z}=0 \text {. }
$$

Then, by using the solution (1.12) of the general exact $\mu$-holomorphy equation, one cane verify that the function

$$
T_{Z Z} \equiv \lambda^{-2} f_{z z}
$$

is holomorphic in the projective structure $(Z, \bar{Z})$, that is

$$
\partial_{\bar{Z}} T_{Z Z}=0
$$


Hence the transition $T_{z z} \rightarrow T_{Z Z}$ transforms the deformed $\mu$-holomorphy condition in the reference complex structure $(z, \bar{z})$ for $T_{z z}$ into the exact $\mu$-holomorphy condition in the same complex structure for $f_{z z}$ and then into the holomorphic condition for $T_{Z Z}$ in the new complex structure $(Z, \bar{Z})$, that is the transform of the complex structure $(z, \bar{z})$ by a quasiconformal transformation. Moreover this transition can be seen as the transformation law of the energy-momentum tensor with respect to the quasiconformal mapping.

Now the combination of equations (2.1), (2.2) and (2.6) enables us to get the general solution of the quantum conformal Ward identity and to express the quantum energymomentum tensor in terms of the classical one as follows:

$$
T_{z z}(z, \bar{z})=\Theta_{z z}(z, \bar{z})+\zeta_{z}(Z) .
$$

This latter relation shows that, at the quantum level, the diffeomorphism anomaly (the quantum corrections of an effective two-dimensional model) is encoded in the Schwarzian derivative of the quasiconformal transformation that transforms a reference complex structure into a projective one and hence can appear as it can disapear depending on the basis we choose.

\section{$3 \mu$-Holomorphic projective connections}

Besides complex structures on the Riemann surface $\Sigma$ there are projective structures which are parametrized by projective connections. for example a holomorphic projective connection $R_{0}$ on the surface $\Sigma$ is an assignment, to any coordinate $z$ of a reference complex structure, of a smooth function $R_{0}$ defined in the domains of $z$ and $z^{\prime}$ by the following transformaion law:

$$
R_{0 z^{\prime} z^{\prime}}^{\prime}\left(z^{\prime}\right)=\left(\partial^{\prime} z\right)^{2}\left[R_{0 z z}(z)-\zeta_{z}\left(z^{\prime}\right)\right]
$$

where $\zeta_{z}\left(z^{\prime}\right) \equiv \partial^{2} \ln \partial z^{\prime}-\frac{1}{2}\left(\partial \ln \partial z^{\prime}\right)^{2}$ is the Schwarzian derivative of the function $z^{\prime}$ with respect to the variable $z$. Further there exists a Beltrami function $\mu$ on the surface $\Sigma$ such that the projective connection $R$ is $\mu$-holomorphic [2]:

$$
W_{2} R=\partial^{3} \mu \text {. }
$$

This means that, to any element $R$ of the space of all projective connections satisfying this equation, there is canonically associated a projective structure subordinated to the conformal structure which is parametrized by a Beltrami function $\mu$ In particular one can deduce from equation (3.2) (by putting $\mu=0$ ) that, in the reference complex structure, the $\mu$-holomorphic projective connection becomes holomorphic. In aonther words a quasiconformal mapping transforms, in a reference complex structure, a holomorphic projective connection into a $\mu$-holomorphic one and conversely:

$$
\begin{aligned}
& R_{0}(z) \stackrel{\mu \neq 0}{\longrightarrow} R(z, \bar{z}), \\
\bar{\partial} R_{0}= & 0 \rightarrow \bar{\partial} R=L_{3}^{R}(\mu),
\end{aligned}
$$

where $L_{3}^{R}$ is the third Bol's operator introduced above. This latter equation can be interpreted as a deformed $\mu$-holomorphy condition for the function $R$. Indeed equation 
(3.2) shows that $R$ is not a tensor (not a 2-differential in the sense of the above definition introduced above). However, by using the $\mu$-holomorphy equation for the Schwarzian derivative $\zeta_{z}(Z)$ (2.6), we get a holomorphic projective connection $R_{Z Z}$ in the complex structure $(Z, \bar{Z}), \partial_{\bar{Z}} R_{Z Z}=0$. This is given by

$$
R_{Z Z}=\lambda^{-2}\left(R_{z z}-\zeta_{z}(Z)\right) \text {. }
$$

Indeed one can verify that the function $R_{Z Z}$ is the coefficient of a 2-differential $(R=$ $R_{Z Z} d Z d Z$ ) and satisfies the following exact $\mu$-holomorphic condition:

$$
W_{0} R_{Z Z}=0
$$

Then a general solution of the $\mu$-holomorphy equation (3.2) is given by

$$
R_{z z}(z, \bar{z})=\zeta_{z}(Z)+f_{z z}
$$

such that $f_{z z}$ is a 2 -differential:

$$
W_{0} f_{z z}=0 \text {. }
$$

Moreover its explicit expression as a Neumann series in terms of the Beltrami differential $\mu$ was given in [5].

\section{Conclusion}

Here we have given a geometrical interpretation of the conformal covariance of a twodimensional conformal model. Indeed we have expressed this conformal covariance as an exact $\mu$-holomorphic condition: the deformation of this latter implies the disappearance of this covariance. Moreover we have shown that this latter can be maintained even at the quantum level, but in a special complex basis that is the transform of the reference one by a quasiconformal mapping. On the other hand we have shown that the transformation law of a holomorphic projective connection under a holomorphic change of coordinates is analogous to the transformation law of a $\mu$-holomophic projective connection under a quasiconformal transformation. This holomorphic projective connection is necessary, at the quantum level, to get manifest the conformal covariance of a bidimensional conformal model. Indeed the Polyakov action that is the effective action of the two-dimensional conformal model was expressed in Ref. [7] in terms of this projective connection.

\section{References}

[1] Witten E, Superstring Theory, Vol. II, Cambridge University Press, Cambridge, 1987.

[2] Zucchini R, Comm. Math. Phys. 152 (1993), 269.

[3] Lazzarini S, Doctoral thesis, LAPP Annecy-Le-Vieux, France, 1990.

[4] Kachkachi M, Dafounansou O and El Rhalami A, Mod. Phys. Lett. A 15 (2000), 417.

[5] Kachkachi M and Kessabi M, J. Phys. A: Math. Gen. 34 (2001), 1.

[6] Kachkachi M, J. Geo. Phys. 40 (2001), 56.

[7] Kachkachi M, Phys. Lett. B 490 (2000), 242. 